\title{
Vascular Endothelial Glycocalyx as a Mechanism of Vascular Endothelial Dysfunction and Atherosclerosis
}

\author{
Minako Yamaoka-Tojo ${ }^{1,2}$ \\ ${ }^{1}$ Department of Rehabilitation/Regenerative Medicine and Cell Design Research Facility, School of Allied Health Sciences, \\ Kitasato University, Sagamihara, Japan \\ ${ }^{2}$ Department of Cardiovascular Medicine, Graduate School of Medical Sciences, Kitasato University, Sagamihara, Japan \\ Email: myamaoka@med.kitasato-u.ac.jp
}

How to cite this paper: Yamaoka-Tojo, M. (2020) Vascular Endothelial Glycocalyx as a Mechanism of Vascular Endothelial Dysfunction and Atherosclerosis. World Journal of Cardiovascular Diseases, 10, 731-749. https://doi.org/10.4236/wjcd.2020.1010070

Received: September 9, 2020

Accepted: October 27, 2020

Published: October 30, 2002

Copyright $\odot 2020$ by author(s) and Scientific Research Publishing Inc. This work is licensed under the Creative Commons Attribution International License (CC BY 4.0).

http://creativecommons.org/licenses/by/4.0/

\section{(c) (i) Open Access}

\begin{abstract}
Atherosclerosis occurs as a result of organized processes that include vascular endothelial dysfunction, lipid accumulation, abnormal inflammatory reaction, excessive reactive oxygen species production, and vascular cell proliferation and migration. In patients with atherosclerosis, vascular endothelial dysfunction is commonly observed with the damage of vascular endothelial glycocalyx, which is an extracellular matrix bound to and encapsulating the endothelial cells that line the blood vessel wall. Unhealthy lifestyle choices such as smoking and physical inactivity also induce glycocalyx degradation. Additionally, vascular endothelial glycocalyx can be damaged by various pathological conditions including dehydration, acute infectious disease, trauma, sepsis, acute respiratory distress syndrome, Kawasaki disease, preeclampsia, gestational diabetes mellitus, hypertension, diabetes mellitus, chronic kidney disease, atherosclerosis, stroke, dementia, microvascular angina, acute coronary syndrome, and heart failure. Vascular endothelial glycocalyx has been shown to be important as a physical cytoprotective barrier for vascular endothelial cells and as a regulatory mechanism for intracellular cell signaling. Therefore, vascular endothelial glycocalyx has immense potential in the exploration of novel strategies for the evaluation of beneficial conditions of healthy vasculature.
\end{abstract}

\section{Keywords}

Vascular Endothelial Function, Inflammation, Oxidative Stress, Cardiovascular Disease Prevention, Disease Management 


\section{Vascular Endothelial Cell Dysfunction}

All the blood vessels of the body have a layer of endothelial cells. Vascular endothelial cells are known to produce various physiologically active substances and are responsible for homeostasis in a healthy body. The combined length of blood vessels in an average human is about two and a half times the earth's circumference, and the combined mass of the vascular endothelial cells in an average human is comparable with the mass of the largest human internal organ, which is the liver. Vascular endothelial cells play various roles in the body such as in inflammation/anti-inflammation, coagulation/fibrinolysis system, and promotion/inhibition of angiogenesis, and they ensure homeostasis by regulating balances (Figure 1). Healthy vascular endothelial cells show good functional properties. Vascular endothelial dysfunction is mainly caused by coronary risk factors such as smoking, hypertension, diabetes, dyslipidemia, obesity, and physical inactivity, and these factors promote atherosclerosis progression (Figure 2). Comprehensive disease management including smoking cessation and control of lifestyle-related diseases can help maintain good vascular endothelial function.

Vascular endothelial function test is used as a predictor of the onset of future cardiovascular events and to determine the effectiveness of comprehensive disease management in cardiovascular medicine. Although it is said that "humans grow older with blood vessels," vascular endothelial function is an integrated index that indicates the health of blood vessels, and maintaining good vascular endothelial function benefits general health. It is necessary to conduct more molecular-level research to determine the exact mechanisms of vascular endothelial cell function.

\section{Endothelial Glycocalyx and Atherosclerosis}

The barrier of extracellular polymeric compounds consists of glycoproteins that form a $200-500 \mathrm{~nm}$ glycosylated gel layer that covers vascular endothelial cells.

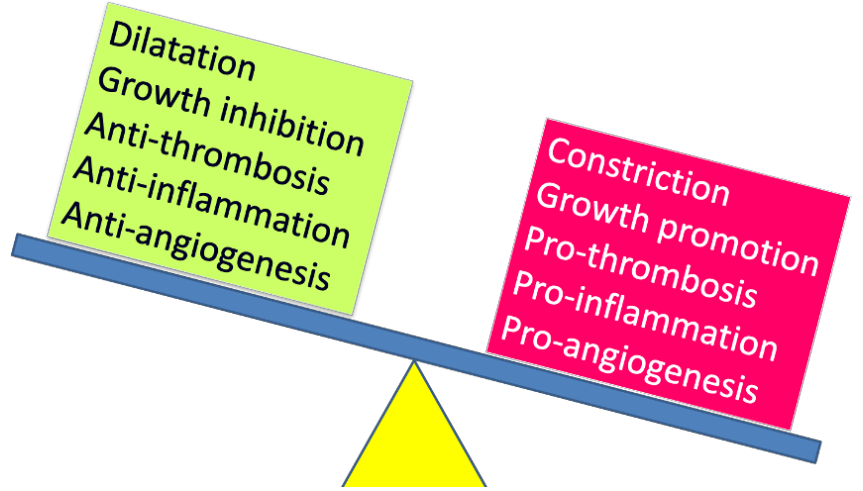

Figure 1. Vascular endothelial dysfunction. Vascular endothelial dysfunction equals vascular endothelial cell dysfunction that leads to vascular failure such that the balance of good vascular conditions cannot be maintained. 


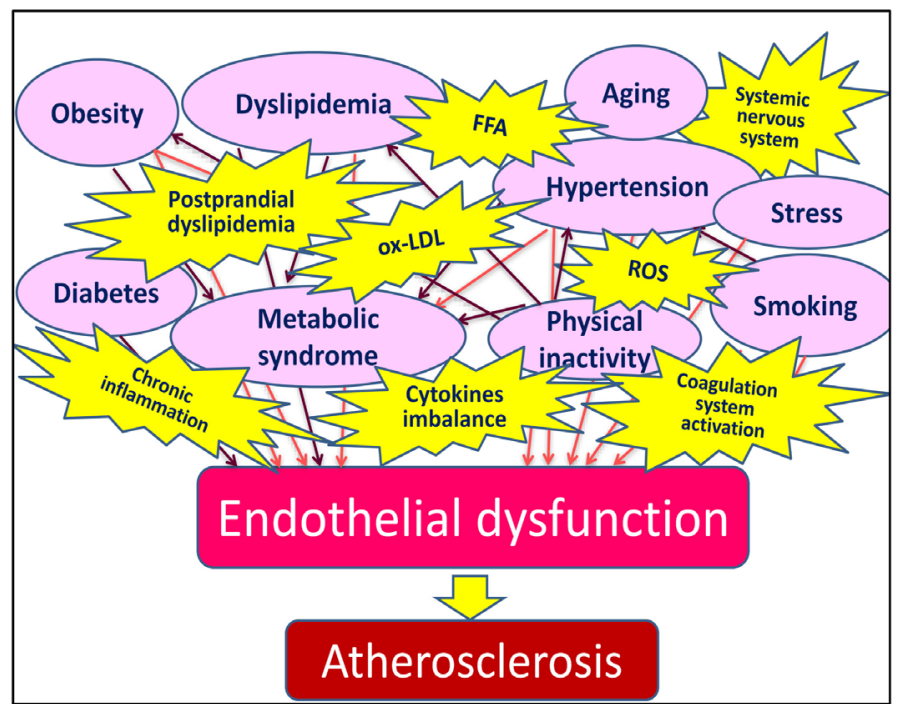

Figure 2. Endothelial dysfunction induces atherosclerosis progression. Vascular endothelial function may have prognostic value for early detection of atherosclerosis. FFA, fatty free acids; ROS, reactive oxygen species, Ox-LDL, oxidized low-density lipoprotein.

The gel layer is called the vascular endothelial glycocalyx. In a healthy vascular endothelial cell covering with enough glycocalyx, the cells are well organized and have good vascular endothelial functions including production of nitric oxide (NO), anticoagulant factors, and sufficient reactive oxygen species (ROS) for vascular homeostasis (Figure 3(a)).

The glycocalyx is composed of proteoglycans and glycosaminoglycans. The proteoglycans include syndecans such as syndecan-1 (CD138) and syndecan-4, glypican, mimecan, perlecan, and biglycan. The glycosaminoglycans include hyaluronan (hyaluronic acid and hyaluronate), heparin, heparan sulfate, chondroitin, dermatan sulfate, and keratan sulfate. The vascular endothelial glycocalyx plays the important roles of physically covering vascular endothelial cells, maintaining cell-cell adhesion of vascular endothelial cells, and controlling cell signaling between vascular endothelial cells, which may affect cancer metastasis, angiogenesis, and vascular permeability. The vascular endothelial glycocalyx is necessary for the maintenance of vascular endothelial cell function and homeostasis [1].

It is involved in the control of intracellular signal transduction (including the proliferation, differentiation, and apoptosis of vascular endothelial cells), cytoskeleton, thrombus formation, inflammatory/anti-inflammatory control, and immunity. Attention has been focused on the relationship between vascular endothelial glycocalyx and the pathogenesis of arteriosclerosis. It was suggested that the condition of the vascular endothelial glycocalyx is associated with the progression of atherosclerotic diseases such as plaque formation and progression, thrombus formation, and plaque rupture and with the pathogenesis of cardiovascular events. The vascular endothelial glycocalyx can be damaged by 


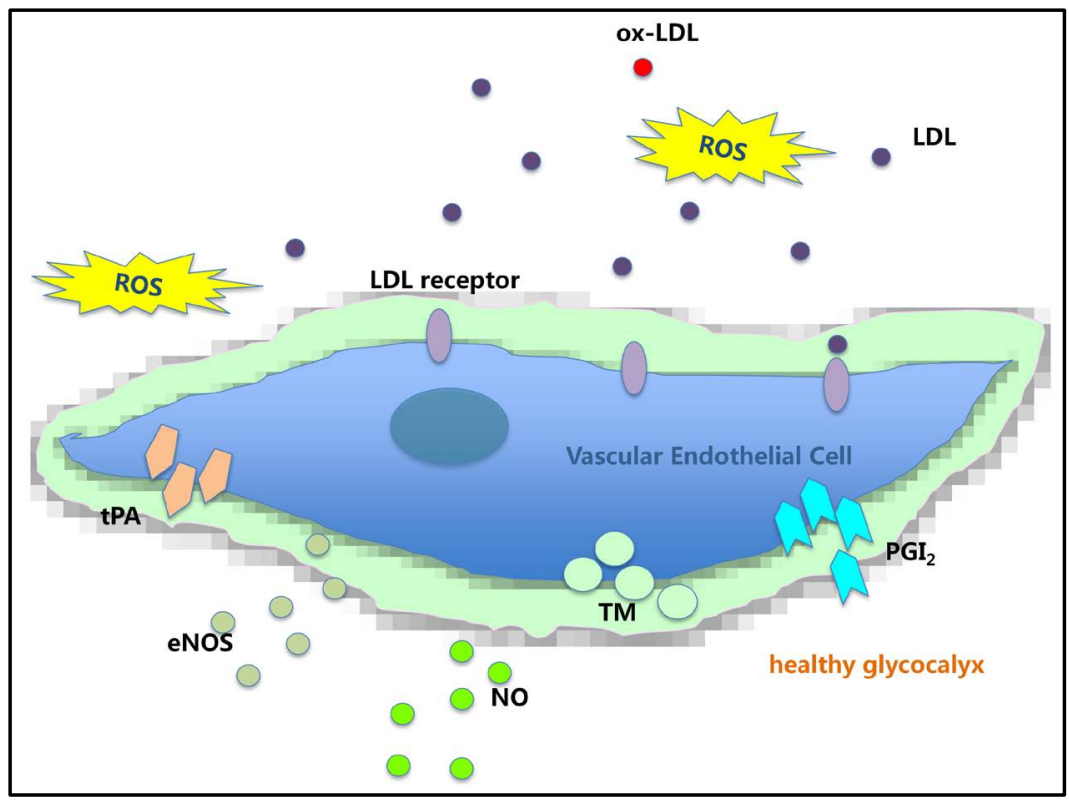

(a)

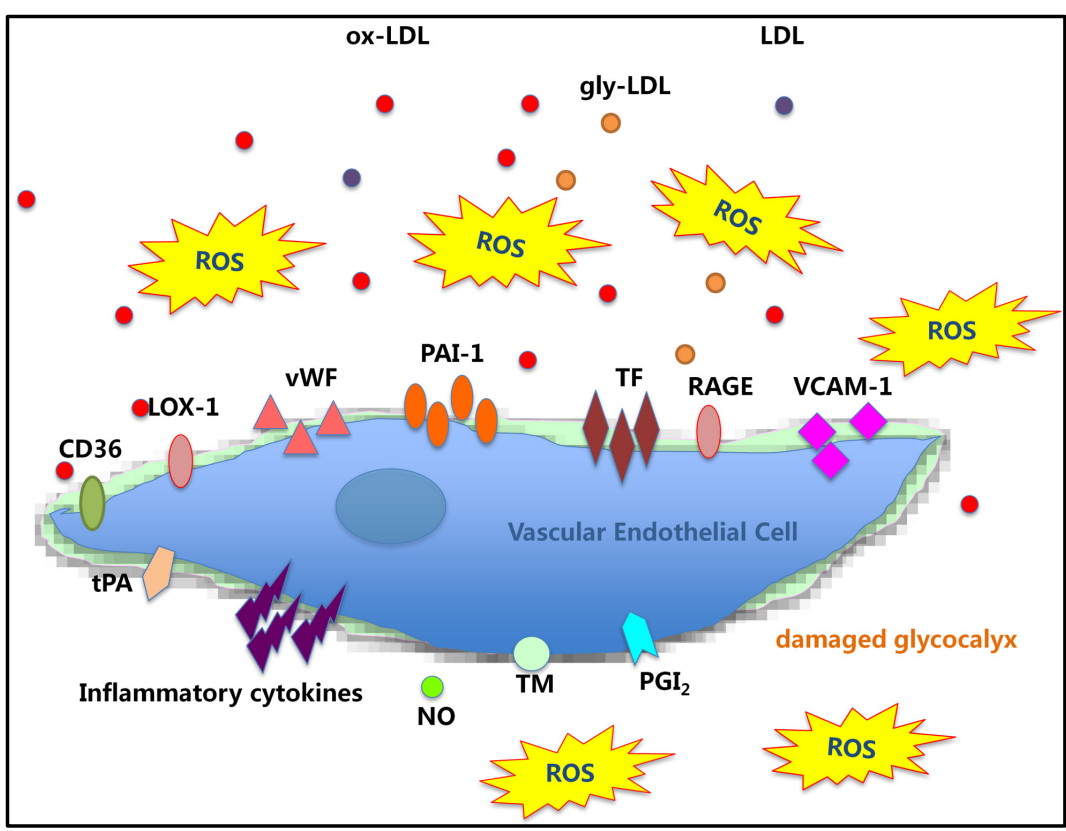

(b)

Figure 3. Vascular endothelial glycocalyx covering vascular endothelial cells. (a). a healthy vascular endothelial cell covering with enough glycocalyx. Endothelial cells covered with glycocalyx are well maintained and adequately perform their function of producing nitric oxide (NO), anticoagulant factors, and sufficient reactive oxygen species (ROS) for vascular homeostasis. (b). an unhealthy vascular endothelial cell partly covered with thinned glycocalyx. Vascular endothelial cells covered with vascular endothelial glycocalyx damaged by hyperglycemia or oxidized LDL produce excessive ROS and low NO, which promote inflammatory cytokine release and coagulation. LOX-1, lectin-like oxidized LDL receptor; VCAM-1, vascular cell adhesion molecule-1; PAI-1, plasminogen activator inhibitor-1; TF, tissue factor; vWF, von Willebrand factor; ox-LDL, oxidized low-density lipoprotein; eNOS, endothelial NO synthase, MMPs, matrix metalloproteases; tPA, tissue plasminogen activator; PGI2, prostacyclin; TM, thrombomodulin. 
endotoxins, inflammation, hypernatremia [2], oxidized low-density lipoprotein (LDL) [3], hyperglycemia, excessive fluid infusion, dehydration, and reduced vascular wall shear stress. These stimuli systemically induce shedding of vascular endothelial glycocalyx, and the glycocalyx layer is thinned under these conditions (Figure 3(b)).

In vascular endothelial cells partly covered by damaged endothelial glycocalyx, high amounts of inflammatory cytokines are released, excessive ROS are produced, and NO production is reduced, which promotes atherosclerosis progression. As shown in Table 1, vascular endothelial glycocalyx is associated with various pathological conditions. It is important to note that vascular endothelial glycocalyx damage has been reported in patients with diabetes [4] [5], smoking [6], obesity [7], hypertension [8], inflammatory disease [9], Kawasaki disease [10] [11], sepsis [12] [13] [14], ischemia/reperfusion injury [15], trauma [16], acute lung injury/acute respiratory distress syndrome [17], acute kidney injury [18], preeclampsia [19], familial hypercholesterolemia [20], chronic kidney disease (CKD) [21], acute coronary syndrome [22] [23], coronary artery disease [24], chronic heart failure [25], microvascular angina [26], stroke [27], and cardiogenic shock [28]. It has been suggested that vascular endothelial glycocalyx damage is involved in the onset and deterioration of these diseases. In these

Table 1. Summary of vascular endothelial glycocalyx and related diseases.

\begin{tabular}{|c|c|c|}
\hline Disease category & Conditions & References \\
\hline \multirow{9}{*}{ Risk factors } & Diabetes & $4,5,52$ \\
\hline & Smoking & 6 \\
\hline & Obesity & 7 \\
\hline & Hypertension & $6,8,59$ \\
\hline & Dyslipidemia & 6,8 \\
\hline & Familial hypercholesterolemia & 20 \\
\hline & CKD & $21,53,54$ \\
\hline & Hemodialysis & 55 \\
\hline & Dementia & 62 \\
\hline \multirow{9}{*}{ Acute disease } & Trauma & 16 \\
\hline & Sepsis & $12-14$ \\
\hline & Ischemia/reperfusion injury & 15 \\
\hline & ALI/ARDS & 17 \\
\hline & Kawasaki disease & 10,11 \\
\hline & Inflammatory disease & 9 \\
\hline & Preeclampsia & 19 \\
\hline & Hemorrhagic shock & 7 \\
\hline & Acute kidney injury & 18 \\
\hline \multirow{7}{*}{ Cardiovascular disease } & Acute coronary syndrome & $22,23,56$ \\
\hline & Coronary artery disease & 58,59 \\
\hline & Chronic heart failure & 25 \\
\hline & Microvascular angina & 26 \\
\hline & Cardiogenic shock & 28 \\
\hline & Stroke & 27,61 \\
\hline & Atherosclerosis progression & 53,59 \\
\hline
\end{tabular}

CKD, chronic kidney disease; ALI/ARDS, acute lung injury/acute respiratory distress syndrome. 
patients, the fragmented vascular endothelial glycocalyx is detected in high concentrations in blood and urine, and the vascular endothelial glycocalyx layer is thinned. Additionally, a coarse and fragile glycocalyx layer resulting from vascular endothelial glycocalyx damage is commonly observed in these patients. Furthermore, by improving the pathological condition through therapeutic intervention, vascular endothelial glycocalyx damage can be ameliorated. Therefore, research on vascular endothelial glycocalyx aimed at the prevention of disease onset and exacerbation and at new therapeutic strategies is ongoing.

It was observed that the vascular endothelial glycocalyx is damaged from the early stage of arteriosclerosis because of exposure to coronary risk factors such as smoking, hypertension, diabetes, and dyslipidemia [29]. Reportedly, vascular endothelial glycocalyx improves dramatically as a result of lifestyle changes such as smoking cessation [30]. Vascular endothelial glycocalyx damage is also caused by a decrease in shear stress, and it is known that vascular endothelial glycocalyx damage leads to vulnerable plaque formation in areas of arterial bifurcation and strong flexion. In vivo and in vitro models showed that decreased shear stress causes vascular endothelial glycocalyx degradation, and plaque formation was strongly induced at the same site [31].

\section{Molecular Biological Mechanism of Vascular Endothelial Glycocalyx Damage in Arteriosclerosis}

As shown in animal models and in humans, arteriosclerosis induces vascular endothelial glycocalyx damage with accompanying elevation in the blood levels of syndecan-1 and hyaluronan. Increased glycocalyx damage results in further thinning of the vascular endothelial glycocalyx layer. It is known that reduced shear stress also causes thinning of vascular endothelial glycocalyx through inactivation of adenosine monophosphate-activated protein kinase and activation of hyaluronidase 2, which induces shedding of hyaluronan and exfoliates vascular endothelial glycocalyx [31]. Syndecan-1 is a molecule known to regulate shear stress-related signals. In syndecan-1-deficient mice, signals that induce inflammation and arteriosclerosis are activated, vascular permeability is enhanced, and leukocyte cell adhesion on vascular endothelial cells is activated [32]. However, it has been confirmed that these signals are suppressed by the re-expression of syndecan-1, and the progression of arteriosclerosis is inhibited. Syndecan-1 is deeply involved in pathological conditions such as vascular hyperpermeability associated with acute inflammation and is used in clinical studies as a major biomarker of vascular endothelial glycocalyx damage.

Factors that directly damage vascular endothelial glycocalyx include reactive oxygen and nitrogen species, uric acid [33], oxidized LDL [3], matrix metalloproteinases (MMPs), heparanase, and sialidase, and these stimuli reduce vascular endothelial function. Administration of 4-methylumbelliferone, a hyaluronan synthesis inhibitor, to high-fat diet-fed ApoE knockout mice resulted in impaired vascular endothelial glycocalyx from the early stage, increased blood 
pressure, decreased acetylcholine-dependent vasorelaxation, and accelerated aortic plaque formation with increased macrophage accumulation [34].

Conversely, antithrombin, tumor necrosis factor- $\alpha$ inhibitor [35], allopurinol [33], sphingosine-1 phosphate [36], MMP inhibitors, hyaluronic acid, and steroids have been reported to protect the vascular endothelial glycocalyx. As these substances are considered to have anti-inflammatory and anti-oxidative effects, they suppress inflammation and excessive oxidative stress in vascular endothelial cells and in the vascular endothelial glycocalyx. It is also possible that these substances directly protect the vascular endothelial glycocalyx.

In a morphological study of ApoE knockout mice, a high-fat diet-induced arteriosclerosis [37] has been reported. The vascular endothelial glycocalyx layer covering the plaque site was found to thin significantly in mice with arteriosclerosis induced with a high-fat diet. However, even in mice with atherosclerosis, vascular endothelial glycocalyx at plaque-free sites was not thinned. The thickness of the vascular endothelial glycocalyx in these mice was found to be comparable with that in normal high-fat diet-fed control mice [37]. Furthermore, the rate of vascular endothelial cell apoptosis at plaque-forming sites was found to be higher than that at non-plaque-forming sites, and shedding of the glycocalyx, promotion of plaque formation, and high expression of MMP-9 were simultaneously detected at the same sites. Vascular endothelial glycocalyx may contribute to plaque stabilization and maintenance of good vascular endothelial function, and its damage may play an essential role in triggering plaque rupture in patients with acute coronary syndrome.

Hyaluronic acid, which is one of the important components of vascular endothelial glycocalyx, and its binding protein, $\mathrm{CD} 44$, are strongly expressed in atherosclerotic lesions. The hyaluronic acid/CD44 signal activates vascular smooth muscle migration and proliferation, which promote plaque formation and increase intercellular matrix synthesis [29]. Furthermore, in the mechanism underlying atherosclerosis induction by hyaluronic acid, hyaluronic acid activates Toll-like receptors (TLRs) to enhance the accumulation of macrophages in atherosclerotic lesions and promote T-helper 1 cell polarization, thereby promoting macrophage immunity and inflammatory response through these cells [38]. Conversely, hyaluronic acid, which abounds in the vascular endothelial glycocalyx layer, inhibits adhesion of leukocytes and platelets to vascular endothelial cells and controls inflammatory reactions in the extracellular matrix. Hyaluronic acid is known to have antithrombotic and anti-inflammatory properties and may suppress atherosclerosis progression by targeting hyaluronan synthase and its receptors [39] [40].

Biglycan, a member of the class I family of small leucine-rich proteoglycans, abounds in the vascular endothelial glycocalyx layer and atherosclerotic plaque. Circulating biglycan is high in patients with acute coronary syndrome and was found in atherosclerotic plaques in ApoE knockout mice. In ApoE/biglycan double knockout mice, thrombin, platelets, and adhesion molecules were acti- 
vated, and the level of inflammatory cytokines and macrophage accumulation in the aortic wall increased [41]. Therefore, loss of biglycan is considered to be closely involved in atherosclerosis progression and plaque formation, especially in the thinning layer of the vascular endothelial glycocalyx.

\section{Vascular Endothelial Function and Glycocalyx}

In the very early stage of atherosclerosis, vascular endothelial function is reduced by exposure to coronary risk factors such as hypercholesterolemia, hyperglycemia, hypertension, obesity, excessive oxidative stress, and chronic inflammation. Vascular endothelial dysfunction is observed in patients with early-stage atherosclerosis and in those with heart failure and hypertension. Therefore, vascular endothelial function attracts attention as an independent prognostic factor of cardiovascular disease. Before vascular endothelial dysfunction, vascular endothelial glycocalyx damage accompanied by a decrease in endothelium-dependent vasodilation reaction and increased vascular permeability occurs. Therefore, the vascular endothelial glycocalyx is considered a major determinant of vascular endothelial function and can be measured by the perfused boundary region (PBR) of sublingual microcirculation.

In an atherosclerosis model using ApoE/LDL receptor-deficient mice, an increased level of biomarkers associated with vascular endothelial dysfunction was detected as a pre-stage of atherosclerotic plaque formation [42]. Specifically, circulating levels of vascular endothelial-origin biomarkers such as the fragmented vascular endothelial glycocalyx syndecan-1 and endothelialcell specific molecule-1, soluble vascular adhesion molecule-1 (sVCAM-1), the coagulation/fibrinolysis system factor known as von Willebrand factor (vWF), tissue-type plasminogen activator, plasminogen activator inhibitor-1, vascular hyperpermeability-related molecule of soluble fms-like tyrosine kinase-1 (sFlt-1), and angiopoietin-2 increased. Decreased endothelium-dependent vasodilation, increased vascular endothelial permeability, and decreased NO production were observed from the very early onset of arteriosclerosis (i.e., at 4 weeks after birth). In the evaluation of vascular endothelial function by flow-mediated dilation (FMD) using magnetic resonance imaging and of vascular endothelial glycocalyx using atomic force microscopy, vascular endothelial dysfunction, thinning of the glycocalyx layer, and detachment of the vascular endothelial glycocalyx were observed after the appearance of microscopic abnormalities in vascular endothelial function. This multistep vascular endothelial damage contributes to atherosclerotic plaque formation, and a more detailed examination of the vascular endothelial glycocalyx-related mechanism is underway.

\section{Atherosclerotic Risk Factors and Vascular Endothelial Glycocalyx}

Cardiovascular diseases are mainly based on atherosclerosis progression induced by coronary risk factors such as smoking, hypertension, dyslipidemia, diabetes, 
and obesity (Figure 4). These risk factors induce endothelial dysfunction, oxidative stress, coagulation cascade activation, inflammation, and accumulation of lifestyle diseases to stimulate further atherosclerosis progression. Lifestyle change is one of the main approaches to improve these risk factors and prevent cardiovascular disease. The main objectives of total risk management for the prevention of cardiovascular disease progression in patients with atherosclerosis are to reduce the rate of cardiovascular events, prevent chronic heart failure, and improve quality of life.

This section presents recent important findings regarding the relationship between each coronary risk factor and vascular endothelial glycocalyx (Figure 5).

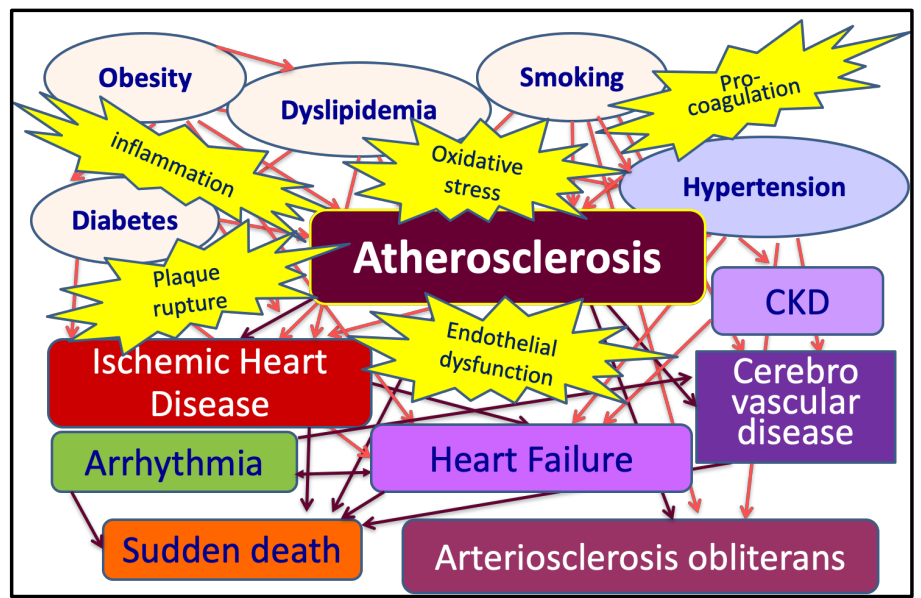

Figure 4. Risk factors of atherosclerosis progression and cardiovascular disease. Cardiovascular diseases are mainly caused by atherosclerosis progression induced by coronary risk factors such as smoking, hypertension, dyslipidemia, diabetes, and obesity. These risk factors induce endothelial dysfunction, oxidative stress, coagulation cascade activation, and inflammation, and these kinds of morbidity promote atherosclerosis progression. CKD, chronic kidney disease.

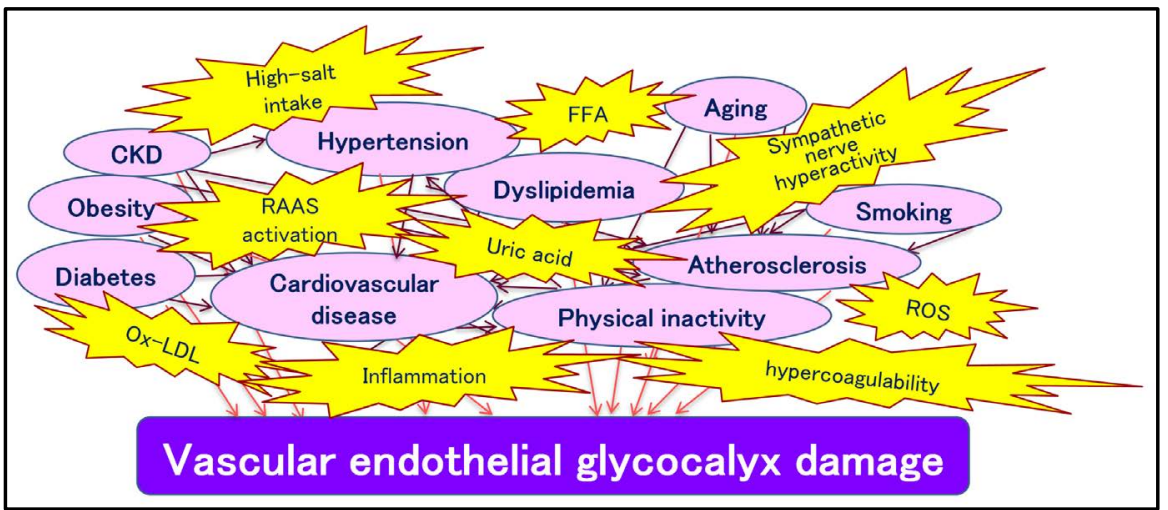

Figure 5. The mechanism for vascular endothelial glycocalyx damage. Vascular endothelial glycocalyx is damaged by cardiovascular diseases and coronary risk factors such as smoking, hypertension, dyslipidemia, diabetes, and obesity. CKD, chronic kidney disease; FFA, free fatty acids; ROS, reactive oxygen species, RAAS, renin-angiotensin-aldosterone system; ox-LDL, oxidized low-density lipoprotein. 


\subsection{Diabetes}

In a hyperglycemic mouse model, hyaluronan, a component of vascular endothelial glycocalyx, was shown to be reduced, and this resulted in increased vascular permeability [43]. In a hyperglycemic model using human microvascular arterial endothelial cells (HMAECs), hyperglycemia was found to activate TLR-2/4 signals, which induce inflammation, promote leukocyte adhesion to HMAECs, reduce heparan sulfate expression, and increase the release of hyaluronic acid [44]. These signals were shown to increase the generation of excessive ROS. Conversely, antioxidants were shown to reduce TLR-2/4 gene expression and downstream inflammatory biomarkers. Furthermore, the vascular endothelial glycocalyx of retinal blood vessels of diabetic model rats has been reported to be significantly thinned compared with that of control rats [45].

The effectiveness of antidiabetic drugs is reported in several animal models and experiments using cultured human cells. In a diabetic mice model, metformin administration for 2 weeks was shown to improve systemic vascular endothelial glycocalyx barrier function even when the blood glucose level was not affected [46]. In recent years, sodium-glucose transporter (SGLT)-2 inhibitors have been reported to have cardio-/reno-protective effect. It was also reported that the incidence of cardiovascular death and exacerbation of heart failure reduced. Empagliflozin, a SGLT-2 inhibitor, improves glycemic control and reduces cardiovascular death and hospitalization due to heart failure. In a study that used cultured human abdominal aortic vascular endothelial cells, empagliflozin has been reported to have a protective effect on vascular endothelial glycocalyx [47]. The protective effect of SGLT-2 inhibitors on vascular endothelial glycocalyx is considered as one of the important mechanisms of these drugs in cardiovascular disease prevention.

\subsection{Dyslipidemia}

Oxidized LDL is considered to be a strong inducer of atherosclerosis progression, and its mechanism of action is direct vascular endothelial glycocalyx damage [3]. It has been reported in animal studies that approximately $60 \%$ of the vascular endothelial glycocalyx is shed off the surface of vascular endothelial cells $25 \mathrm{~min}$ after the administration of oxidized LDL, and at the same time, the activation of platelet adhesion is observed on the vascular wall [3]. A clinical study on the estimated whole-body vascular endothelial glycocalyx volume reported a significant decrease in patients with familial hypercholesterolemia compared with that in healthy subjects [20]. The volume of vascular endothelial glycocalyx was partially improved with statin therapy but was significantly lower in patients with familial hypercholesterolemia than that in healthy subjects.

\subsection{Hypertension}

Similar to diabetes and dyslipidemia, hypertension damages the vascular endothelial glycocalyx. Reportedly, the vascular endothelial glycocalyx in the retina 
and choroid of hypertensive rats significantly degraded and were exfoliated from the blood vessels and leukocyte and platelet adhesion to vascular endothelium accelerated at sites where vascular endothelial glycocalyx had thinned [45]. In cultured vascular smooth muscle cells, glycocalyx covering the cell membrane was found to be damaged by high-salt loading in 2 - 4 days, but vascular smooth muscle remodeling and cell hypertrophy were observed 6 - 16 days after continuous high-salt loading [48]. Since proliferation and hypertrophy of vascular smooth muscle cells are deeply involved in the progression of hypertension and arteriosclerosis, salt reduction aimed at the vascular endothelial glycocalyx may be an effective treatment for patients with hypertension.

Although excessive salt intake causes salt-sensitive hypertension, the negatively charged vascular endothelial glycocalyx neutralizes cell surface charges by retaining sodium in the plasma in the glycocalyx layer. Furthermore, the vascular endothelial glycocalyx has been reported to be a regulator of salt sensitivity [49]. Salt reduction is considered a preventive or therapeutic measure against hypertension as too much salt induces myocardial cell hypertrophy even under conditions of stable blood pressure, and the secretion of aldosterone from the adrenal gland results in hypertension and causes hypertrophy and fibrosis of the heart and blood vessels. Furthermore, excessive salt intake has been determined to increase urinary excretion of potassium and calcium, which may cause renal stones and osteoporosis, and potassium deficiency increases blood pressure. Although further studies are necessary, the hypernatremia-induced vascular endothelial glycocalyx damage and activation of vascular smooth muscle remodeling should be considered in the determination of the mechanism of hypertension development and arteriosclerosis progression.

\subsection{Chronic Kidney Disease (CKD)}

The involvement of albuminuria in the early stage of diabetic nephropathy has been identified as clearance of negatively charged albumin increases due to vascular endothelial glycocalyx damage [50]. Reportedly, glomerular vascular endothelial glycocalyx damage can be caused by hyperglycemia, diabetic nephropathy, or adriamycin administration [51]. In a study on gestational diabetes mellitus, the patient group with a higher rate of vascular endothelial glycocalyx release in the blood had a higher probability of developing diabetes mellitus in the future [52]. Based on these findings, the onset and exacerbation of renal damage in various diseases can be predicted from measurements of blood levels of the fragmented and released vascular endothelial glycocalyx.

Syndecan-1 is produced by vascular endothelial cells and glomerular epithelial cells (podocytes), and it is associated with impaired barrier function during glomerular filtration. In patients with CKD of stages 3 - 5, increasing circulating syndecan- 1 and hyaluronan levels are associated with decreasing vascular endothelial function and increasing levels of biomarkers related to vascular endothelial damage (sFlt-1, sVCAM-1, vWF, and angiopoietin-2). Furthermore, 
thinning of vascular endothelial glycocalyx and increasing levels of syndecan-1 were observed in a rat 5/6 nephrectomized CKD model [21].

As vascular endothelial dysfunction occurs in the early stage of glomerular disease, arteriosclerosis may continue to progress and the rate of cardiovascular death may increase [53]. A cross-sectional study of patients with initial nephrotic syndrome reported a significant increase in circulating syndecan-1 levels in patients who retained renal function [54]. Vascular endothelial function measured by FMD was significantly lower in the patient groups than that in healthy subjects, and the increasing levels of syndecan-1 were closely related to vascular endothelial dysfunction measured by FMD. Thus, vascular endothelial glycocalyx damage accompanied by the release of the constituent syndecan- 1 and vascular endothelial dysfunction is assumed to occur at almost the same time.

Dialysis treatment may become necessary for patients with advanced renal dysfunction. However, dialysis treatment is a risk factor that strongly promotes arteriosclerosis. PBR of sublingual microvessels, which is an important indicator of vascular endothelial glycocalyx damage, was enlarged in patients who underwent dialysis therapy. Furthermore, circulating hyaluronan and syndecan-1 levels were increased, and the activation of hyaluronidase was enhanced in patients who underwent dialysis therapy [55]. Vascular endothelial glycocalyx damage was strongly observed in dialysis patients with high levels of inflammatory biomarkers. This suggests that atherosclerosis progression and carcinogenesis may be the mechanisms underlying decreased immune function in dialysis patients.

\section{Cardiovascular Diseases and Vascular Endothelial Glycocalyx}

\subsection{Ischemic Heart Disease}

Circulating syndecan-1 levels in 141 patients with acute coronary syndrome were significantly higher than those in 45 patients with chest pain syndrome without coronary artery lesions and those in 24 healthy subjects, and the levels were significantly higher in male patients than those in female patients [22]. The high levels of syndecan-1 in patients with acute coronary syndrome were also significant after adjusting for sex, age, or present and past smoking [22]. Additionally, circulating syndecan-1 levels were useful in the diagnosis of acute coronary syndrome in these patients. However, the blood level of syndecan-4 has been reported to be significantly higher in female patients with acute myocardial infarction [56]. Based on these findings, the circulating syndecan-1 level may be considered a diagnostic biomarker of destabilized coronary plaque.

Compared with the rapid increase in circulating fragmented glycocalyx levels in acute diseases, circulating fragmented glycocalyx levels in patients with chronic pathological conditions such as stable angina were low [57]. In such pathological conditions, thinning of the glycocalyx layer and reduction of the blood level of fragmented glycocalyx occur simultaneously because of the de- 
creased production and accelerated degradation of the vascular endothelial glycocalyx. Hence, the blood glycocalyx level is not considered a suitable prognosis predictor or severity index in chronic disease conditions.

In sublingual microvessels, vascular endothelial glycocalyx thinned according to the severity of atherosclerotic lesions in coronary arteries, and circulating levels of hyaluronic acid in coronary arteries were significantly high in patients with coronary atherosclerosis [58]. In patients with ischemic heart disease and patients at high risk of the disease, PBR was identified as a risk factor of arteriosclerotic cerebrovascular disease and ischemic heart disease, and PBR was found to correlate with systolic blood pressure [59]. High PBR was observed in patients with arteriosclerotic diseases and in patients at high risk of atherosclerotic diseases, and PBR measurement may be effective in disease management and screening of cardiovascular diseases. Expectations for the usefulness of PBR measurement as a judgment index are growing.

\subsection{Cerebrovascular Disease}

Atherosclerotic cerebrovascular disease is one of the age-related diseases of the central nervous system. It is related to vascular endothelial dysfunction and is considered to be the main cause of dementia. PBR was found to be significantly higher in patients with lacunar infarction and white matter lesions than that in healthy individuals or patients without white matter lesions [60]. In a time-course analysis of endothelium-related biomarkers (chondroitin sulfate, heparin sulfate, keratan sulfate, hyaluronic acid, CD44, syndecan-1, syndecan-2, syndecan-3, syndecan-4, glypican-1, and biglycan), plasma concentrations of CD44 were significantly correlated with stroke severity in patients with ischemic stroke, and this finding suggests that a relationship exists between CD44 level, brain tissue damage, and infarct size [61]. The measurement of PBR in arteriosclerotic diseases may be useful for the prediction of severity and prognosis, but further studies are necessary.

Hyaluronic acid levels in the cerebrospinal fluid of 46 patients with cerebrovascular dementia were found to be significantly higher than those of 45 patients with Alzheimer's disease and 26 healthy individuals without dementia [62]. Based on the correlation between hyaluronic acid level in cerebrospinal fluid and vascular endothelial glycocalyx damage in cerebral blood vessels, it was suggested that vascular endothelial glycocalyx damage in the microcirculation of an organ is closely related to the damage of the organ.

\section{How to Reduce the Vascular Endothelial Glycocalyx Damage}

In order to vascular endothelial glycocalyx in a healthy state, basic and clinical studies should be needed to clarify the impaired factors of the glycocalyx in more detail to prevent cardiovascular disease progression. Moreover, large-scale clinical studies may be favorable to determinebenefit factors for healthy vascular en- 
dothelial glycocalyx for cardiovascular prevention. Benefit factors that have been identified at this time include smoking cessation, bodyweight loss in obesity, and moderate exercise. To prevent vascular endothelial glycocalyx damage, it is important to reduce excessive salt intake and too much alcohol drinking and manage lifestyle-related diseases such as diabetes, hypertension, and dyslipidemia inappropriate conditions. Furthermore, it is expected to develop medications that have positive effects on vascular endothelial glycocalyx aside from sleep quality and stress management.

\section{Final Note}

It has been said that vascular endothelial glycocalyx has a primitive barrier function. However, the gel-like component consisting of macromolecules such as sugar chains and glycoproteins flexibly changes their density and composition according to the health condition of the living organism. Vascular endothelial glycocalyx encapsulates various substances, provides various molecules for homeostasis, and regulates the signals of vascular endothelial cells. Although the direct investigation of its in vivo functions is complicated, vascular endothelial glycocalyx has great potential in the exploration of novel strategies for the evaluation of the beneficial conditions of healthy vasculature.

\section{Acknowledgements}

This work was partly supported by JSPS KAKENHI Grants number JP19K11371.

\section{Conflicts of Interest}

The author declares no conflicts of interest regarding the publication of this paper.

\section{References}

[1] Van den Berg, B.M., Vink, H. and Spaan, J.A. (2003) The Endothelial Glycocalyx Protects against Myocardial Edema. Circulation Research, 92, 592-594.

https://doi.org/10.1161/01.RES.0000065917.53950.75

[2] Rorije, N.M.G., Rademaker, E., Schrooten, E.M., et al. (2019) High-Salt Intake Affects Sublingual Microcirculation and Is Linked to Body Weight Change in Healthy Volunteers: A Randomized Cross-Over Trial. Journal of Hypertension, 37, 1254-1261. https://doi.org/10.1097/HJH.0000000000002015

[3] Vink, H., Constantinescu, A.A. and Spaan, J.A. (2000) Oxidized Lipoproteins Degrade the Endothelial Surface Layer: Implications for Platelet-Endothelial Cell Adhesion. Circulation, 101, 1500-1502. https://doi.org/10.1161/01.CIR.101.13.1500

[4] Broekhuizen, L.N., Lemkes, B.A., Mooij, H.L., et al. (2010) Effect of Sulodexide on Endothelial Glycocalyx and Vascular Permeability in Patients with Type 2 Diabetes Mellitus. Diabetologia, 53, 2646-2655. https://doi.org/10.1007/s00125-010-1910-X

[5] Groen, B.B., Hamer, H.M., Snijders, T., et al. (2014) Skeletal Muscle Capillary Density and Microvascular Function Are Compromised with Aging and Type $2 \mathrm{Di}$ abetes. Journal of Applied Physiology, 116, 998-1005.

https://doi.org/10.1152/japplphysiol.00919.2013 
[6] Triantafyllidi, H., Benas, D., Schoinas, A., et al. (2020) Sex-Related Associations of High-Density Lipoprotein Cholesterol with Aortic Stiffness and Endothelial Glycocalyx Integrity in Treated Hypertensive Patients. Journal of Clinical Hypertension (Greenwich). https://doi.org/10.1111/jch.14002

[7] Diebel, L.N., Peraino, N., Westrick, J., et al. (2020) Obesity and Impaired Barrier Function after Shock: A Biomimetic in Vitro Model Using Microfluidics. The Journal of Trauma and Acute Care Surgery, 89, 544-550. https://doi.org/10.1097/TA.0000000000002804

[8] Triantafyllidi, H., Benas, D., Vlachos, S., et al. (2018) HDL Cholesterol Levels and Endothelial Glycocalyx Integrity in Treated Hypertensive Patients. Journal of Clinical Hypertension (Greenwich), 20, 1615-1623. https://doi.org/10.1111/jch.13404

[9] Ostrowski, S.R., Gaini, S., Pedersen, C., et al. (2015) Sympathoadrenal Activation and Endothelial Damage in Patients with Varying Degrees of Acute Infectious Disease: An Observational Study. Journal of Critical Care, 30, 90-96.

https://doi.org/10.1016/j.jcrc.2014.10.006

[10] Ohnishi, Y., Yasudo, H., Suzuki, Y., et al. (2019) Circulating Endothelial Glycocalyx Components as a Predictive Marker of Coronary Artery Lesions in Kawasaki Disease. International Journal of Cardiology, 292, 236-240. https://doi.org/10.1016/j.ijcard.2019.05.045

[11] Luo, L., Feng, S., Wu, Y., et al. (2019) Serum Levels of Syndecan-1 in Patients with Kawasaki Disease. The Pediatric Infectious Disease Journal, 38, 89-94. https://doi.org/10.1097/INF.0000000000002047

[12] Steppan, J., Hofer, S., Funke, B., et al. (2011) Sepsis and Major Abdominal Surgery Lead to Flaking of the Endothelial Glycocalix. Journal of Surgical Research, 165, 136-141. https://doi.org/10.1016/j.jss.2009.04.034

[13] Drost, C.C., Rovas, A., Kusche-Vihrog, K., et al. (2019) Tie2 Activation Promotes Protection and Reconstitution of the Endothelial Glycocalyx in Human Sepsis. Thrombosis and Haemostasis, 119, 1827-1838. https://doi.org/10.1055/s-0039-1695768

[14] Hippensteel, J.A., Uchimido, R., Tyler, P.D., et al. (2019) Intravenous Fluid Resuscitation Is Associated with Septic Endo-Thelial Glycocalyx Degradation. Critical Care, 23, 259. https://doi.org/10.1186/s13054-019-2534-2

[15] Rubio-Gayosso, I., Platts, S.H. and Duling, B.R. (2006) Reactive Oxygen Species Mediate Modification of Glycocalyx during Ischemia-Reperfusion Injury. American Journal of Physiology. Heart and Circulatory Physiology, 290, H2247-H2256. https://doi.org/10.1152/ajpheart.00796.2005

[16] Chignalia, A.Z., Yetimakman, F., Christiaans, S.C., et al. (2016) The Glycocalyx and Trauma: A Review. Shock, 45, 338-348. https://doi.org/10.1097/SHK.0000000000000513

[17] Schmidt, E.P., Overdier, K.H., Sun, X., et al. (2016) Urinary Glycosaminoglycans Predict Outcomes in Septic Shock and Acute Respiratory Distress Syndrome. American Journal of Respiratory and Critical Care Medicine, 194, 439-449. https://doi.org/10.1164/rccm.201511-22810C

[18] Kim, H.B., Soh, S., Kwak, Y.L., et al. (2020) High Preoperative Serum Syndecan-1, a Marker of Endothelial Glycocalyx Degradation, and Severe Acute Kidney Injury after Valvular Heart Surgery. Journal of Clinical Medicine, 9, 1803. https://doi.org/10.3390/jcm9061803

[19] Weissgerber, T.L., Garcia-Valencia, O., Milic, N.M., et al. (2019) Early Onset Preeclampsia Is Associated with Glyco-Calyx Degradation and Reduced Microvascular 
Perfusion. Journal of the American Heart Association, 8, e010647. https://doi.org/10.1161/JAHA.118.010647

[20] Meuwese, M.C., Mooij, H.L., Nieuwdorp, M., et al. (2009) Partial Recovery of the Endothelial Glycocalyx upon Rosuvas-Tatin Therapy in Patients with Heterozygous Familial Hypercholesterolemia. Journal of Lipid Research, 50, 148-153. https://doi.org/10.1194/jlr.P800025-JLR200

[21] Padberg, J.S., Wiesinger, A., di Marco, G.S., et al. (2014) Damage of the Endothelial Glycocalyx in Chronic kidney Disease. Atherosclerosis, 234, 335-343. https://doi.org/10.1016/j.atherosclerosis.2014.03.016

[22] Miranda, C.H., de Carvalho Borges, M., Schmidt, A., et al. (2016) Evaluation of the Endothelial Glycocalyx Damage in Patients with Acute Coronary Syndrome. Atherosclerosis, 247, 184-188. https://doi.org/10.1016/j.atherosclerosis.2016.02.023

[23] Wernly, B., Fuernau, G., Masyuk, M., et al. (2019) Syndecan-1 Predicts Outcome in Patients with ST-Segment Elevation Infarction Independent from Infarct-Related Myocardial Injury. Scientific Reports, 9, Article No. 18367. https://doi.org/10.1038/s41598-019-54937-x

[24] Brands, J., Hubel, C.A., Althouse, A., et al. (2020) Noninvasive Sublingual Microvascular Imaging Reveals Sex-Specific Reduction in Glycocalyx Barrier Properties in Patients with Coronary Artery Disease. Physiological Reports, 8, e14351. https://doi.org/10.14814/phy2.14351

[25] Wadowski, P.P., Hulsmann, M., Schorgenhofer, C., et al. (2018) Sublingual Functional Capillary Rarefaction in Chronic Heart Failure. European Journal of Clinical Investigation, 48, e12869. https://doi.org/10.1111/eci.12869

[26] Jaarsma, C., Vink, H., van Haare, J., et al. (2017) Non-Invasive Assessment of Microvascular Dysfunction in Patients with Microvascular Angina. International Journal of Cardiology, 248, 433-439. https://doi.org/10.1016/j.ijcard.2017.05.010

[27] Ikonomidis, I., Frogoudaki, A., Vrettou, A.R., et al. (2019) Impaired Arterial Elastic Properties and Endothelial Glyco-Calyx in Patients with Embolic Stroke of Undetermined Source. Thrombosis and Haemostasis, 119, 1860-1868.

https://doi.org/10.1055/s-0039-1694752

[28] Marechal, X., Favory, R., Joulin, O., et al. (2008) Endothelial Glycocalyx Damage during Endotoxemia Coincides with Microcirculatory Dysfunction and Vascular Oxidative Stress. Shock, 29, 572-576. https://doi.org/10.1097/shk.0b013e318157e926

[29] Nieuwdorp, M., Meuwese, M.C., Vink, H., et al. (2005) The Endothelial Glycocalyx: A Potential Barrier between Health and Vascular Disease. Current Opinion in Lipidology, 16, 507-511. https://doi.org/10.1097/01.mol.0000181325.08926.9c

[30] Ikonomidis, I., Marinou, M., Vlastos, D., et al. (2017) Effects of Varenicline and Nicotine Replacement Therapy on Arterial Elasticity, Endothelial Glycocalyx and Oxidative Stress during a 3-Month Smoking Cessation Program. Atherosclerosis, 262, 123-130. https://doi.org/10.1016/j.atherosclerosis.2017.05.012

[31] Zhang, J., Kong, X., Wang, Z., et al. (2019) AMP-Activated Protein Kinase Regulates Glycocalyx Impairment and Macrophage Recruitment in Response to Low Shear Stress. FASEB Journal, 33, 7202-7212. https://doi.org/10.1096/fj.201801869RRR

[32] Voyvodic, P.L., Min, D., Liu, R., et al. (2014) Loss of Syndecan-1 Induces a Pro-Inflammatory Phenotype in Endothelial Cells with a Dysregulated Response to Atheroprotective Flow. Journal of Biological Chemistry, 289, 9547-9559. https://doi.org/10.1074/jbc.M113.541573

[33] Ko, J., Kang, H.J., Kim, D.A., et al. (2019) Uric Acid Induced the Phenotype Transi- 
tion of Vascular Endothelial Cells via Induction of Oxidative Stress and Glycocalyx Shedding. FASEB Journal, fj201901148R. https://doi.org/10.1096/fj.201901148R

[34] Nagy, N., Freudenberger, T., Melchior-Becker, A., et al. (2010) Inhibition of Hyaluronan Synthesis Accelerates Murine Atherosclerosis: Novel Insights into the Role of Hyaluronan Synthesis. Circulation, 122, 2313-2322. https://doi.org/10.1161/CIRCULATIONAHA.110.972653

[35] Nieuwdorp, M., Meuwese, M.C., Mooij, H.L., et al. (2009) Tumor Necrosis Factor-Alpha Inhibition Protects against Endotoxin-Induced Endothelial Glycocalyx Perturbation. Atherosclerosis, 202, 296-303. https://doi.org/10.1016/j.atherosclerosis.2008.03.024

[36] Mensah, S.A., Cheng, M.J., Homayoni, H., et al. (2017) Regeneration of Glycocalyx by Heparan Sulfate and Sphingosine 1-Phosphate Restores Inter-Endothelial Communication. PLOS ONE, 12, e0186116. https://doi.org/10.1371/journal.pone.0186116

[37] Cancel, L.M., Ebong, E.E., Mensah, S., et al. (2016) Endothelial Glycocalyx, Apoptosis and Inflammation in an Atheros-Clerotic Mouse Model. Atherosclerosis, 252, 136-146. https://doi.org/10.1016/j.atherosclerosis.2016.07.930

[38] Mullick, A.E., Tobias, P.S. and Curtiss, L.K. (2005) Modulation of Atherosclerosis in Mice by Toll-Like Receptor 2. Journal of Clinical Investigation, 115, 3149-3156. https://doi.org/10.1172/JCI25482

[39] Fischer, J.W. (2019) Role of Hyaluronan in Atherosclerosis: Current Knowledge and Open Questions. Matrix Biology, 78-79, 324-336.

https://doi.org/10.1016/j.matbio.2018.03.003

[40] Grandoch, M., Bollyky, P.L. and Fischer, J.W. (2018) Hyaluronan: A Master Switch Between Vascular Homeostasis and Inflammation. Circulation Research, 122, 1341-1343. https://doi.org/10.1161/CIRCRESAHA.118.312522

[41] Grandoch, M., Kohlmorgen, C., Melchior-Becker, A., et al. (2016) Loss of Biglycan Enhances Thrombin Generation in Apolipoprotein E-Deficient Mice: Implications for Inflammation and Atherosclerosis. Arteriosclerosis, Thrombosis, and Vascular Biology, 36, e41-e50. https://doi.org/10.1161/ATVBAHA.115.306973

[42] Bar, A., Targosz-Korecka, M., Suraj, J., et al. (2019) Degradation of Glycocalyx and Multiple Manifestations of Endothelial Dysfunction Coincide in the Early Phase of Endothelial Dysfunction before Atherosclerotic Plaque Development in Apolipoprotein E/Low-Density Lipoprotein Receptor-Deficient Mice. Journal of the American Heart Association, 8, e011171. https://doi.org/10.1161/JAHA.118.011171

[43] Zuurbier, C.J., Demirci, C., Koeman, A., et al. (2005) Short-Term Hyperglycemia Increases Endothelial Glycocalyx Permeability and Acutely Decreases Lineal Density of Capillaries with Flowing Red Blood Cells. Journal of Applied Physiology, 99, 1471-1476. https://doi.org/10.1152/japplphysiol.00436.2005

[44] Pahwa, R., Nallasamy, P. and Jialal, I. (2016) Toll-Like Receptors 2 and 4 Mediate Hyperglycemia Induced Macrovascular Aortic Endothelial Cell Inflammation and Perturbation of the Endothelial Glycocalyx. Journal of Diabetic Complications, 30, 563-572. https://doi.org/10.1016/j.jdiacomp.2016.01.014

[45] Kumase, F., Morizane, Y., Mohri, S., et al. (2010) Glycocalyx Degradation in Retinal and Choroidal Capillary Endothelium in Rats with Diabetes and Hypertension. Acta Medica Okayama, 64, 277-283.

[46] Eskens, B.J., Zuurbier, C.J., van Haare, J., et al. (2013) Effects of Two Weeks of Metformin Treatment on Whole-Body Glycocalyx Barrier Properties in db/db Mice. Cardiovascular Diabetology, 12, 175. https://doi.org/10.1186/1475-2840-12-175 
[47] Cooper, S., Teoh, H., Campeau, M.A., et al. (2019) Empagliflozin Restores the Integrity of the Endothelial Glycocalyx in Vitro. Molecular and Cellular Biochemistry, 459, 121-130. https://doi.org/10.1007/s11010-019-03555-2

[48] Bkaily, G., Simon, Y., Menkovic, I., et al. (2018) High Salt-Induced Hypertrophy of Human Vascular Smooth Muscle Cells Associated with a Decrease in Glycocalyx. Journal of Molecular and Cellular Cardiology, 125, 1-5. https://doi.org/10.1016/j.yjmcc.2018.10.006

[49] Oberleithner, H. and Wilhelmi, M. (2015) Vascular Glycocalyx Sodium Store-Determinant of Salt Sensitivity? Blood Purification, 39, 7-10. https://doi.org/10.1159/000368922

[50] Jeansson, M., Granqvist, A.B., Nystrom, J.S., et al. (2006) Functional and Molecular Alterations of the Glomerular Barrier in Long-Term Diabetes in Mice. Diabetologia, 49, 2200-2209. https://doi.org/10.1007/s00125-006-0319-Z

[51] Jeansson, M., Bjorck, K., Tenstad, O., et al. (2009) Adriamycin Alters Glomerular Endothelium to Induce Proteinuria. Journal of the American Society of Nephrology, 20, 114-122. https://doi.org/10.1681/ASN.2007111205

[52] Long, D.S., Hou, W., Taylor, R.S., et al. (2016) Serum Levels of Endothelial Glycocalyx Constituents in Women at 20 Weeks' Gestation Who Later Develop Gestational Diabetes Mellitus Compared to Matched Controls: A Pilot Study. BMJ Open, 6, $\mathrm{e} 011244$.

[53] Watts, G.F., Herrmann, S., Dogra, G.K., et al. (2001) Vascular Function of the Peripheral Circulation in Patients with Nephrosis. Kidney International, 60, 182-189. https://doi.org/10.1046/j.1523-1755.2001.00785.x

[54] Salmito, F.T., de Oliveira Neves, F.M., Meneses, G.C., et al. (2015) Glycocalyx Injury in Adults with Nephrotic Syndrome: Association with Endothelial Function. Clinica Chimica Acta, 447, 55-58. https://doi.org/10.1016/j.cca.2015.05.013

[55] Vlahu, C.A., Lemkes, B.A., Struijk, D.G., et al. (2012) Damage of the Endothelial Glycocalyx in Dialysis Patients. Journal of the American Society of Nephrology, 23, 1900-1908. https://doi.org/10.1681/ASN.2011121181

[56] Solbu, M.D., Kolset, S.O., Jenssen, T.G., et al. (2018) Gender Differences in the Association of Syndecan-4 with Myocar-Dial Infarction: The Population-Based Tromso Study. Atherosclerosis, 278, 166-173. https://doi.org/10.1016/j.atherosclerosis.2018.08.005

[57] Nemoto, T., Minami, Y., Yamaoka-Tojo, M., et al. (2020) Endothelial Glycocalyx and Severity and Vulnerability of Coronary Plaque in Patients with Coronary Artery Disease. Atherosclerosis, 302, 1-7. https://doi.org/10.1016/j.atherosclerosis.2020.04.014

[58] Xue, X.J., Jiang, Y., Chen, L., et al. (2018) Relationship between the Endothelial Glycocalyx and the Extent of Coronary Atherosclerosis. Microcirculation, 25, e12504. https://doi.org/10.1111/micc.12504

[59] Gorshkov, A.Y., Klimushina, M.V., Boytsov, S.A., et al. (2018) Increase in Perfused Boundary Region of Endothelial Glycocalyx Is Associated with Higher Prevalence of Ischemic Heart Disease and Lesions of Microcirculation and Vascular Wall. Microcirculation, 25, e12454. https://doi.org/10.1111/micc.12454

[60] Martens, R.J., Vink, H., van Oostenbrugge, R.J., et al. (2013) Sublingual Microvascular Glycocalyx Dimensions in Lacunar Stroke Patients. Cerebrovascular Diseases, 35, 451-454. https://doi.org/10.1159/000348854

[61] DellaValle, B., Hasseldam, H., Johansen, F.F., et al. (2019) Multiple Soluble Components of the Glycocalyx Are Increased in Patient Plasma after Ischemic Stroke. 
Stroke, 50, 2948-2951. https://doi.org/10.1161/STROKEAHA.119.025953

[62] Nagga, K., Hansson, O., van Westen, D., et al. (2014) Increased Levels of Hyaluronic Acid in Cerebrospinal Fluid in Patients with Vascular Dementia. Journal of Alzheimer's Disease, 42, 1435-1441. https://doi.org/10.3233/JAD-141200 